\title{
Discrete dislocation dynamics analysis of the effect of lattice orientation on void growth in single crystals
}

\author{
Javier Segurado, Javier LLorca
}

Departmento de Ciencia de Materiales, Universidad Politécnica de Madid G instituto Madirleño de Estudios Avanzados de Materiales (iMDEA-Materiales), E.TS de ingenieros de Caminos, 28040 Madrid, Spain

Keymords:

Dislocations

Numerical simulations

Plastic deformation

Single crystals

\begin{abstract}
A B S T R A C T
The micromechanisms of plastic deformation and void growth were analyzed using discrete dislocation dynamics in an isolated FCC single crystal deformed in-plane strain in the (1 10 ) plane. Three different stress states (uniaxial tension, uniaxial deformation and biaxial deformation) were considered for crystals oriented in different directions and with a different number of active slip systems. It was found that strain hardening and void growth rates depended on lattice orientation in uniaxial tension because of anisotropic stress state. Crystal orientation did not influence, however, hardening and void growth when the crystals were laaded under uniaxial or biaxial deformation because the stress state was more homogeneous, although both (hardening and void growth rates) were much higher than under uniaxial tension. In addition, the number of active slip systems did not substantially modify the mechanical behavior and the void growth rate if plastic deformation along the available slip systems was compatible with overall crystal deformation prescribed by the boundary conditions. Otherwise, the incompatibility between plastic deformation and boundary conditions led to the development of large hydrostatic elastic stresses, which increased the strain hardening rate and reduced the void growth rate.
\end{abstract}

\section{Introduction}

The overall ductility of metals is mainly controlled by the ability of the material to withstand the growth of micron-sized voids up to the point at which fracture is triggered by the sudden coalescence of neighbor voids into a crack. Microvoids normally appear within a grain (single crystal) and their growth is driven by plastic flow. The importance of this phenomenon to understand and control ductile fracture has long been recognized and many analytical and numerical models of void growth have been developed over the years (Rice and Tracey, 1969; Gurson (1977); Hom and McMeeking, 1989; Worswick and Pick, 1990; Koplik, 1988; Tvergaard, 1990 among many others). These models were based on standard isotropic plasticity formulations and did not take into account two important factors: the anisotropic nature of plastic flow in single crystals and the size effects that appear when plastic flow is confined into very small regions (Fleck et al., 1994; Uchic et al., 2004; Motz et al., 2005).

The influence of plastic anisotropy and crystal orientation on void deformation and growth has been analyzed using crystal plastic models within the framework of the finite element model (Quinn et al., 1995; Gan et al., 2006; Potirniche et al., 2006b; Liu et al., 2007) or slip line theory (Kysar et al., 2005). These analyses concluded that crystal orientation did influence void growth rate under uniaxial tension but this effect diminished rapidly as triaxiality increased (Potirniche et al., 2006b). In parallel, other investigations addressed the issue of size effect within the framework of isotropic, strain gradient plasticity 
theories (Liu et al., 2003; Liu et al., 2005; Li and Steinmann, 2006; Srinivasan et al., 2008), and consistently predicted a reduction in void growth rate for smaller voids. Finally, the combined effect of lattice orientation and void size has only been analyzed very recently through the application of strain gradient plasticity models within the framework of single crysta] plasticity (Borg et al., 2008). This investigation confirmed that void growth rates decreased with void size and that lattice orientation did not significantly influence void growth rates under high triaxiality conditions. Nevertheless, quantitative estimations of void growth rates and cavitation stress depend on the magnitude of intrinsic length scale included in the strain gradient plasticity formulation, which has to be obtained by fitting to experimental results. Reliable experimental data on growth rates for micro-sized voids are not available, to the authors' knowledge, and so the accuracy of these quantitative predictions remains unknown.

Void growth in single crystals can be also studied using other simulation strategies, like molecular dynamics (Potirniche et al., 2006a; Traiviratana et al., 2008). However, the scope of these analyses was limited to understand the physical mechanisms of void growth by dislocation emission due to the inherent spatial and temporal limitations of this methodology. Another modeling technique that naturally accounts for both the effect of the plastic anisotropy and the plasticity size effect is discrete dislocation dynamics (DDD). The length scale that controls the size effect is incorporated without any fitting parameter through the length of the Burgers vector, while plastic deformation by dislocation motion along slip planes is the very essence of the technique. DDD has been used to study plastic deformation patterns around a rectangular void according to lattice orientation for different slip system orientations (Hussein et al., 2008) and more recently to analyze the effect of the void size on the growth rate for circular void within an infinite crystal (Huang et al., 2007) or micron-size single crystals (Segurado and LLorca, 2009). A clear size effect of the type "smaller is slower" was found when the crystals were deformed in uniaxial and biaxial deformation, and the size effect also increased with the level of triaxial stresses. This behavior was expected and predicted by strain gradient plasticity models but it was confirmed for the first time in these DDD simulations, which include the physical mechanisms of void growth. ln addition, the simulation results in terms of void growth rates and plastic deformation patterns could be used as benchmarks for calibrating strain gradient plasticity models, which can then be used as quantitative tools to predict ductile fracture in metallic materials.

In this paper, the DDD framework in Segurado and Llorca (2009) is used to assess the effect of crystal orientation on the micromechanisms of plastic deformation and the void growth rate in a FCC single crystal deformed in plane strain in the (1 10) plane. Simulations were carried out under different stress states (uniaxial traction, uniaxial deformation and biaxial deformation) and considered also the influence of the number of active slips systems. Particular emphasis was placed on relating the patterns of plastic deformation with the mechanical behavior of the voided crystal and the void growth rates.

\section{Discrete dislocation dynamics model}

The simulation of the growth of a cylindrical void within a single crystal was carried out within the framework of the DDD strategy developed by Van der Giessen and Needleman (1995). This strategy was extended by Romero et al. (2008) to nonconvex domains through the use of finite elements with embedded discontinuities and it is briefly recalled here. The model considers an elastic, isotropic crystal of dimensions $L \times L$ with a void of radius $R$ at the center (Fig. 1). An FCC lattice is considered and plane strain conditions are assumed in the $x_{1}-x_{2}$ plane, which corresponds to the crystallographic plane ( $\left.\overline{1} 10\right)$. It is known (Rice, 1987) that the twelve slip systems of the FCC crystal can be reduced to three effective in plane slip systems which admit a two-dimensional plane strain deformation state under the action of loads contained in the $(\overline{1} 10)$ plane. The

L

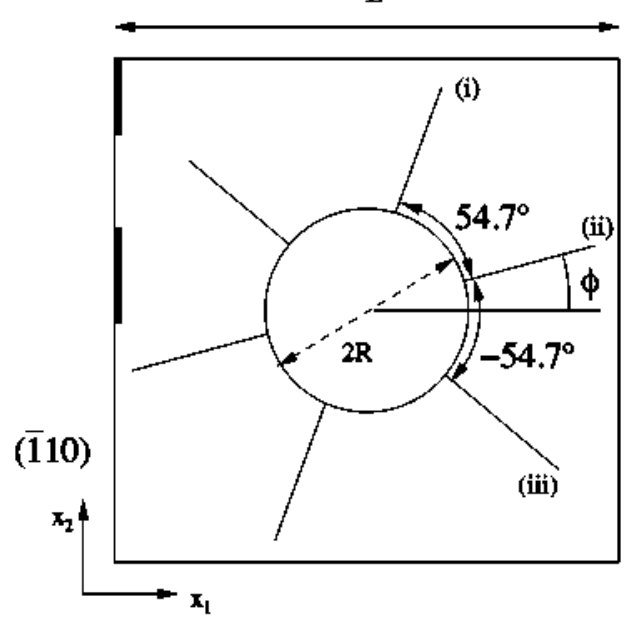

Fig. 1. Scheme of the boundary value problem for the analysis of void growth in a single crystal subjected to plane strain deformation in the (i 10$)$ plane. 
three effective slip systems are shown in Fig. 1 (more details can be found in Kysar et al. (2005)). System (ii) stands for the complex slip system parallel to $[\overline{1} 10]$, while systems (i) and (iii) - that form angles of $54.7^{\circ}$ and $-54.7^{\circ}$ with respect to sys-

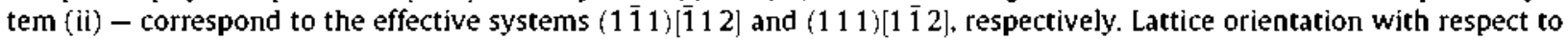
the $x_{1}-x_{2}$ reference frame is expressed by the angle $\phi$ that the slip system (ii) forms with the $x_{1}$ direction. This nomenclature will be followed in this paper to indicate the lattice orientation with respect to the main loading axis $x_{2}$.

Dislocations within the 2D ctystal are represented by linear singularities perpendicular to the crystal plane with Burgers vector b whose modulus is given by $b$. Dislocation dipoles can be nucleated at discrete points randomly distributed on the slip planes, which mimic the behavior of Frank-Read sources in 2D, the Burgers vectors tb being parallel to the slip plane direction. Nucleation occurs when the magnitude of the resolved shear stress at the source $\tau$ exceeds a critical value $\tau_{\text {nuc }}$ over a period of time $t_{\text {nuc }}$. The distance between the two new dislocations, $L_{\text {nuc }}$, is given by

$$
L_{\text {nuc }}=\frac{E}{4 \pi(1-v)(1+v)} \frac{b}{\tau_{\text {ruc }}}
$$

so that the resolved shear stress balances the attractive forces between them.

Once generated, dislocations slip in their respective glide planes, and the speed $v_{i}$ of dislocation $i$ is given by

$$
v_{\mathrm{i}}=\frac{\tau_{i} b}{\mathrm{~B}}
$$

where $\tau_{i}$ stands for the projection of the Peach-Koehler force acting on the dislocation $i$ in the sliding direction and $B$ is the drag coefficient. Two dislocations of different sign gliding on the same slip plane are annihilated when they cross each other or if they fall within a distance $L_{o n h}=6 b$. This annililation distance was introduced by Van der Giessen and Needleman (1995) to overcome the limitation of continuum mechanics to determine the stress field very close to the dislocation core and it follows the work of Essmann and Mughrabi (1979), who found that two edge dislocations of different sign could not coexist without instantaneously collapsing if the distance between them was of this order. Finally, if a dislocation exits the crystal, the dislocation is deleted from the simulation and a displacement jump of $b / 2$ is introduced along the slip plane.

The shear stress component of the Peach-Koehler force acting on the ith dislocation, located on a glide plane with unit normal $\mathbf{n}$ and unit tangent vector $\mathbf{m}$, can be obtained by projecting the stress at the location of the dislocation according to

$$
\tau_{i}=\mathbf{m} \cdot\left(\partial+\sum_{j \neq i} \partial_{i}^{j}\right) \cdot \mathbf{n} .
$$

where $\hat{\sigma}_{i}^{j}$ stands for the stress due to the $j$ th dislocation and it is computed analytically from the expressions for the stress field induced by an edge dislocation on an infinite, elastic and isotropic continuum. To this field it is necessary to add $\hat{\sigma}$ which includes the effect of the image forces induced by the crystal boundaries on the dislocations. At a given stage of loading, the stress and strain fields in the crystal are obtained by the superposition of the two fields, the first one given by the sum of those induced by the individual dislocations in the current configuration and the second one, which corrects for the actual boundary conditions. This term is computed by solving a linear elastic boundary value problem using the finite element method with the appropriate boundary conditions, as detailed in Van der Giessen and Needleman (1995) and Segurado et al. (2007).

The deformation process of the crystal is solved in an explicit incremental manner, using a Euler forward time-integration algorithm for the equations of motion. Once the new positions of all dislocations at time $t$ have been computed, new dislocations are generated at the sources according to (1) and dislocation pairs of opposite sign in the same slip plane are annihilated when they are within $L_{a n n}$. The boundary conditions for the linear elastic boundary value problem are computed from the new dislocation structure and the applied displacements for time $t+\delta t$. The resolved shear stresses on the dislocations are computed according to (3) from the fields induced by the dislocations and the solution of the boundary value problem. Then, the velocities of the dislocations are obtained from the corresponding resolved shear stress, as given by (2) and the new positions are computed from these velocities. More details about the numerical implementation of the model can be found in Segurado et al. (2007) and Romero et al. (2008). It should be noticed that the current version of the program has been parallelized to improve performance in multiprocessor shared-memory computers.

\section{Results and discussion}

The model described in the foregoing section was applied to simulate the deformation of an isolated square single crysta] of side length $L=2.5 \mu \mathrm{m}$ which contained a cylindrical void at the center. The void radius $R$ was $0.446 \mu \mathrm{m}$, covering $10 \%$ of the crystal area (Fig. 1). The crystal was made up of a linear elastic, isotropic solid, characterized by its shear modulus $\mu=26.32 \mathrm{GPa}$ and Poisson's ratio $\nu=0.33$.

Plastic deformation occurred along the three slip systems (i)-(iii) depicted in Fig. 1 and the distance between the slip planes of each system was taken as $100 b$. Plastic deformation in micron-sized crystals is localized in a very limited number of slip planes containing a dislocation source which is activated upon deformation. Thus, it is not necessary to include all the slip planes in each active slip system in the model. It was shown (Van der Giessen and Needleman, 1995) that the simulation 
results are independent of the separation between slips planes if this numerical parameter is of the order of $100 \mathrm{~b}$. The modulus of the Burgers vector was $0.25 \mathrm{~nm}$ and the slip planes were initially free of dislocations. The drag coefficient $B$, which relates the dislocation speed with the resolved shear stress on the glide plane was $10^{-4}$ Pas. Sources were distributed randomly along the slip planes with density of $150 \mu \mathrm{m}^{-2}$. Previous analyses for this geometry with source densities in the range $75-300 \mathrm{fm}^{-2}$ led to equivalent results because only a fraction of the sources available are in fact active during loading. The critical resolved shear stress for dislocation nucleation in each source was assigned randomly following a Gaussian distribution with an average value of $50 \mathrm{MPa}$ and a standard deviation of $15 \mathrm{MPa}$. The nucleation time for all sources was 0.01 Hs. These magnitudes are similar to those used previously in several investigations (Deshpande et al., 2005; Chng et al., 2006; Segurado et al., 2007; Segurado and Llorca, 2009). There were no obstacles to the motion of dislocations within the crystal but the external crystal boundaries were assumed to be impenetrable to dislocations, and so dislocations could only leave the crystal through the central void. The time step in the Euler forward time-integration algorithm was constant and equal to $0.05 \mathrm{~ns}$ and the maximum velocity of the dislocations was limited to $100 \mathrm{~m} / \mathrm{s}$. The values of these two numerical parameters have been validated in previous studies (Segurado et al., 2007).

The kinetics of microvoid growth was analyzed in plane strain under three loading conditions with different triaxiality: uniaxjal tension, uniaxial deformation and isotropic deformation in the ( 110$)$ plane. The boundary conditions for uniaxial tension along the $x_{2}$-axis are expressed as (Fig. 1 ):

$$
\begin{aligned}
& \mathrm{u}_{2}=0 \text { and } T_{1}=0 \text { on } x_{2}=0 \\
& \mathrm{u}_{2}=U \text { and } T_{1}=0 \text { on } x_{2}=L \\
& T_{1}=T_{2}=0 \text { on } x_{1}=0 \text { and } x_{1}=L
\end{aligned}
$$

while the last equation in (4) is substituted by

$$
u_{1}=0 \text { and } T_{2}=0 \text { on } x_{1}=0 \text { and } x_{1}=L
$$

in the case of uniaxial deformation along the $x_{2}$-axis or by

$$
\begin{aligned}
& u_{1}=0 \text { on } x_{1}=0 \text { and } u_{1}=U \text { on } x_{1}=L \\
& T_{2}=0 \text { on } x_{1}=0 \text { and } x_{1}=L
\end{aligned}
$$

for biaxial deformation. Loading was imposed by applying a constant strain rate of $\dot{\epsilon}=\dot{U} / \mathrm{L}=2000 \mathrm{~s}^{-1}$ up to a maximum strain of $2 \%$.

The normal stress $\sigma_{22}$ acting on the crystal face $x_{2}=L$ was computed by averaging the normal tractions on this face according to

$$
\sigma_{22}=\frac{1}{L} \int_{x_{2}=L} T_{2} \mathrm{~d} x_{1}
$$

and a similar expression was used to obtain the normal stress $\sigma_{11}$ acting on $x_{1}=L$ for uniaxial and biaxial deformation.

\subsection{Effect of Iattice orientation}

The square crystal was loaded along the $x_{2}$-axis to study the mechanical behavior in four different crystal orientations included in plane $(\overline{1} 10)$ : $[001],[\overline{1} 12],[\overline{1} 1,1]$ and $[\overline{1} 10]$. These lattice orientations correspond to a rotation of the slip systems given by $\phi=0^{\circ}, 35.5^{\circ}, 54.7^{\circ}$ and $90^{\circ}$, respectively (Fig. 1). For each orientation, simulations were carried out under unjaxial tension $\left(\sigma_{11}=0\right)$, unaxial deformation $\left(\epsilon_{11}=0\right.$ ) and biaxial deformation $\left(\epsilon_{11}=\epsilon_{22}\right)$ to study the influence of hydrostatic stresses on the mechanical response and void growth rates.

\subsubsection{Mechanical behavior}

The stress-strain curves $\left(\sigma_{22}-\epsilon_{22}\right)$ of the voided crystal deformed in uniaxial tension are plotted in Fig. 2(a). Each curve corresponds to one of the four lattice orientations and is the average of three simulations obtained with different distributions of the sources within the crystal. The evolution of the dislocation density with the applied strain is plotted Fig. 2(b) for each orientation.

The crystals were free of dislocations at the beginning of the analyses and the initial response was linear elastic. The yield point was controlled by the nucleation of first dislocation dipole and it was practically equivalent for all lattice orientations. Nucleation of dislocations in a given slip system $\alpha$ is controlled by the resolved shear stress on the slip plane $\tau^{\alpha}$, which is given by

$$
\tau^{\alpha}=\mathbf{m}^{\alpha} \oslash \mathbf{n}^{\alpha} \cdot \sigma
$$

where $\boldsymbol{\sigma}$ is the stress tensor at the source position and $\mathbf{m} \otimes \mathbf{n}$ is the Schmidt tensor. $\mathbf{m}$ and $\mathbf{n}$ depend only on the cosines of $\phi$ and $90-\phi$ for the three slip systems considered here and the highest Schimdt factor in one of the three slip planes is the same for the four orientations. As the source density is high, there is always a source close to the point in the ciystal with the highest $\sigma$ and the crystals yield at the same macroscopic stress irrespective of the lattice orientation. 

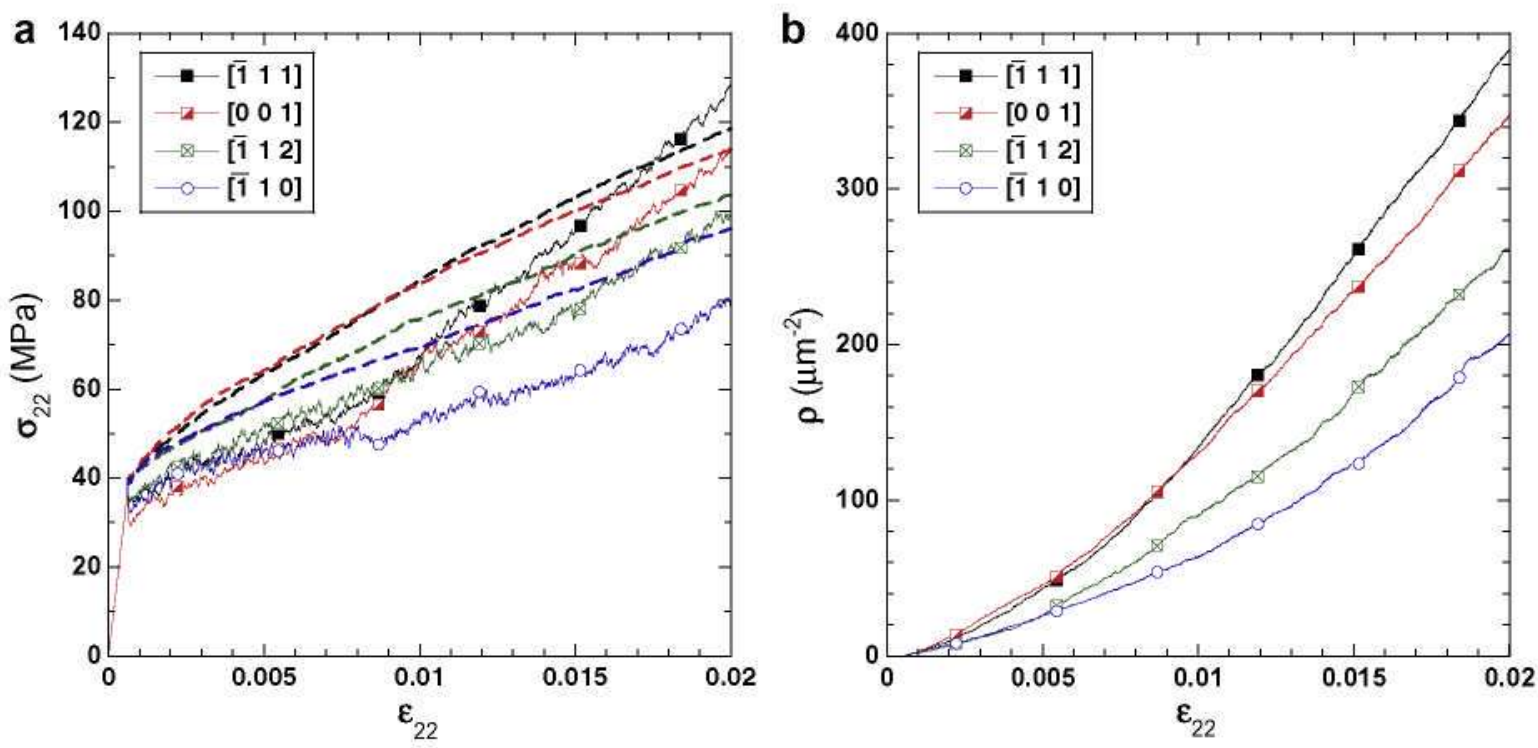

Fig. 2. (a) Averaged $\sigma_{22}-\epsilon_{22}$ curves of the voided crystal under uniaxial traction for the different orientations. The predictions based on the Taylor model are also plotted for comparison. (b) Evolution of the dislocation density with the applied strain.

After yielding, more dislocation dipoles were formed near to the equator of the void (where stresses are maximum) in the slip planes with the highest Schmidt factor. Plastic deformation occurred by dislocation slip which ends when the dislocation reaches the void surface or the crystal boundary. Dislocations exited the crystal in the former case leading to a step on the void surface or they were stopped at the crystal boundaries and formed pile-ups. These pile-ups hindered the mobility of dislocations and generated a back stress which also reduced the activity of the sources. The development of back stresses by the pile-ups at the boundaries and the interaction between the stress fields generated by dislocations in different slip systems (forest hardening) were responsible for the strain hardening of the voided crystals after yielding. These effects were fairly independent of the crystal orientation at small strains (when the dislocation densities are reduced) and the stressstrain curves were initially very close for the different crystal orientations. However, the hardening rates depended on the crystal orientation at higher strains $\left(\epsilon_{22} \geqslant 1 \%\right)$ and were correlated with the dislocation densities (Fig. 2a and b).

A first order estimation of the effect of the differences in dislocation density on the flow stress can be assessed from the application of the Taylor dislocation model (Taylor, 1934, 1938). This model was developed to take into account the effect of forest dislocations in hardening, which predicts that the flow stress is proportional to the square root of the dislocation density. Mott (1952) also found the same functional dependence between the flow stress and the dislocation density when hardening was due to the formation of pile-ups at obstacles, and thus the tensile flow stress can be expressed as

$$
\sigma=\sigma_{y 0}+M \alpha \mu b \sqrt{\rho}
$$

in which $\sigma_{y 0}$ stands for the initial flow stress necessary to nucleate dislocations in the crystal (which was equal for all orientations), $\alpha$ is an empirical material constant whose value is approximately 0.3 (Taylor, 1934, 1938), and $M$ the Taylor factor, which depends on the orientation of the slip planes with respect to loading. For simplicity, $M$ was taken as the highest Schmidt factor among all the slip systems for each orientation. The predictions of Eq. (9) can be found in Fig. 2a, where $\rho$ was the total dislocation density for each orientation (as shown in Fig. 2b). They were in reasonably good agreement with the DDD simulations, taking into account that there are no adjustable parameters in the Taylor model, although it should be noticed that the overestimated the numerical predictions at low strains. Better predictions in this regime could have been obtained using the density of non-mobile dislocations (instead of the total density) in Eq. (9) but this magnitude was not available from the simulations.

The differences in the hardening rates and dislocation densities can be ascertained from the analyses of the contour plots of the total strain and of the dislocation patterns for the orientations [1 10] (Fig. 3) and [111] (Fig. 4) which led to the minimum and maximum hardening rates, respectively. In the crystal loaded in the [110] direction $\left(\phi=90^{\circ}\right)$, the slip systems (i) and (iii) were well-oriented for dislocation motion and had the same Schmidt factor. Plastic deformation was localized in both systems (Fig. 3a), while the activity of the slip system (ii) (parallel to the $x_{2}$-axis) was minimum. Deformation was symmetric and the number of positive and negative dislocations within the crystal was balanced. In addition, many slip planes in the systems (i) and (iii) began at the void and ended at the lateral surface of the crystal $\left(x_{1}=0\right.$ or $\left.L\right)$. It is evident that plastic deformation was localized in these slip planes (Fig. 3a), which were also located in the crystal region where the applied stresses were maximum (they are minimum above and below the void). Thus, the confluence of several factors (high Schmidt factor and applied stresses, free surfaces that attract dislocations at both ends of the slip plane) favored the easy glide of 
a

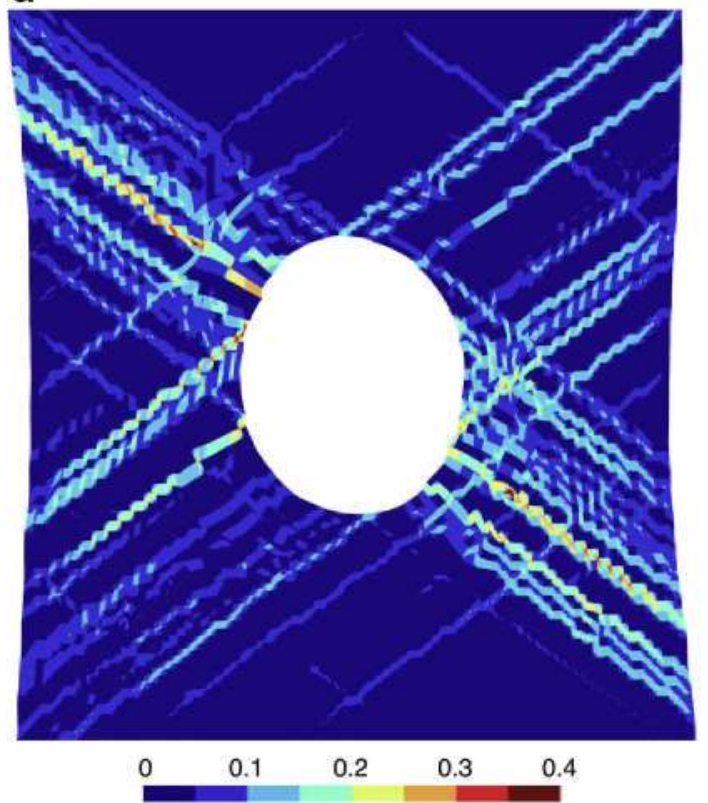

b

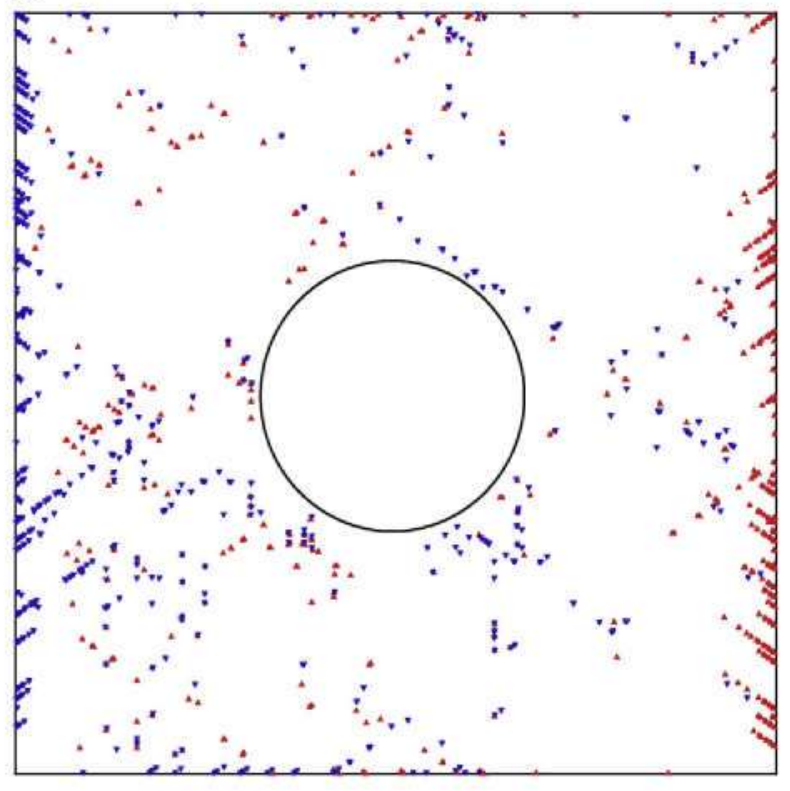

Fig. 3. (a) Contour plot of the total strain in the crystal subjected to uniaxial traction in the [110] direction at a far-field strain of $2 \%$. The displacement magnification factor is equal to 3. (b) Dislocation pattern corresponding to (a). Blue and red symbols stand for dislocations of different sign. (For interpretation of color mentioned in this figure the reader is referred to the web version of the article.)

a

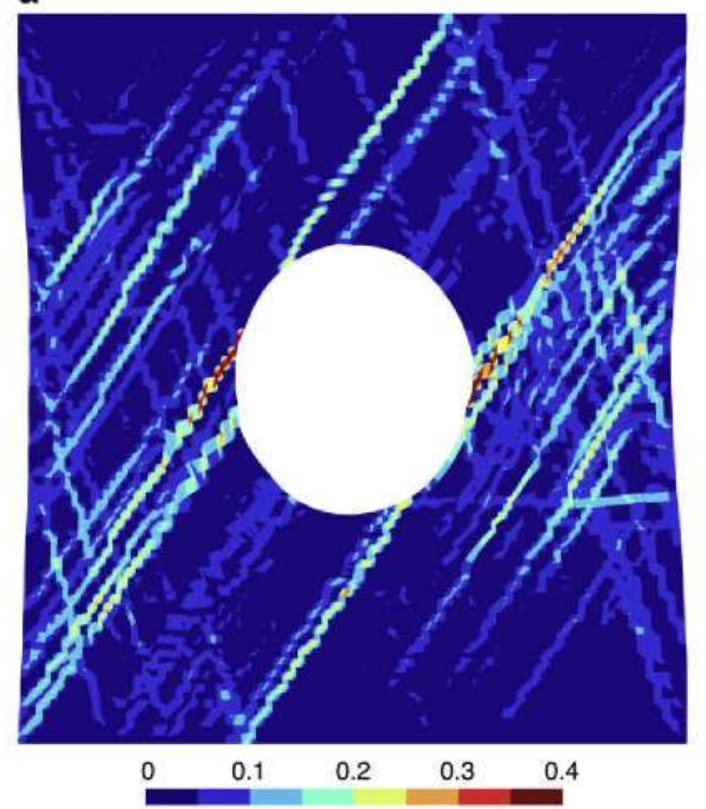

b

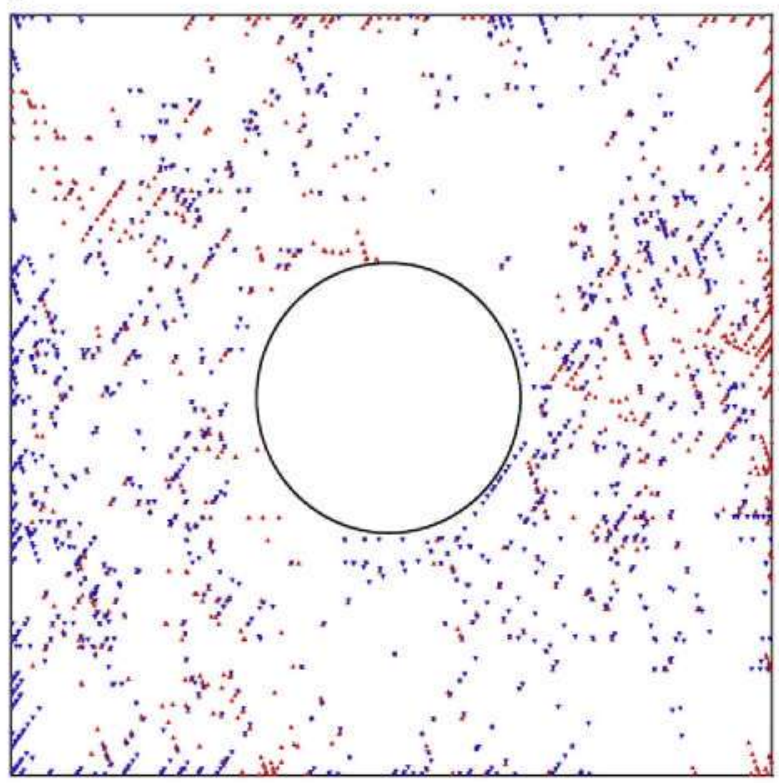

Fig. 4. (a) Contour plot of the total strain in the crystal subjected to uniaxial traction in the [111] direction at a far-field strain of $2 \%$. The displacement magnification factor is equal to 3. (b) Dislocation pattern corresponding to (a). Blue and red symbols stand for dislocations of different sign. (For interpretation of color mentioned in this figure the reader is referred to the web version of the article.)

dislocations in both slip planes at low applied stresses. In fact, as the dislocations could slip easily along systems (i) and (iii) and many slip planes ended in the void surfaces, the dislocations within the crystal were mainly concentrated in pile-ups against the lateral crystal surfaces (Fig. 3b). 
Maximum hardening was observed in the crystal loaded along the [111] direction (Fig. 4). The orientation of the slip systems was given in this case by $\phi=54.7^{\circ}$ and the slip system (ii) had the highest Schmidt factor (equivalent to systems (i) and (iii) in the soft $[\overline{1} 10]$ orientation analyzed above). Most of the dislocation activity was concentrated along this plane because the slip system (iii) was parallel to the $x_{1}$-axis (a thus, its Schimdt factor is 0 ) while the slip system (iii) forms an angle of $70.6^{\circ}$ with the $x_{2}$ and the corresponding Schmidt factor was very low (Fig. 4a). Thus, deformation was not symmetric in this case and localized in slip system (ii), in agreement with the results obtained by Potirniche et al. (2006b) in their finite element simulations of a periodic voided single crystal. Most of the active slip planes in this system ended in the upper or lower crystal surfaces $\left(x_{2}=0\right.$ or $\left.L\right)$, where the boundary conditions impose a fixed displacement. While the stress-free boundary conditions at the lateral and void surfaces attract dislocations, the image forces due to the imposed displacements repel the dislocations and were added to the back-stress generated by the dislocation pile-ups at the crystal boundaries. In addition, the most active slip planes have to pass above and below the void, precisely the region where the stresses coming from the external forces are minimum. As a result of all these factors, higher stresses were necessary to accommodate the imposed displacement by plastic deformation and the difficulties associated with dislocation motion led to a homogeneous distribution of dislocations within the crystal (Fig. 4b). These factors (location and orientation of active slip planes, image forces induced by boundary conditions) also explained the hardening rates observed along the [001] and [112] orientations. The corresponding figures are not included for the sake of brevity.

Uniaxial tension leads to an anisotropic stress state within the voided crystal in which the orientation and location of the slip plane controls its potential to accommodate plastic deformation by dislocation motion. The panorama changes under uniaxial $\left(\epsilon_{11}=0\right)$ or biaxial $\left(\epsilon_{11}=\epsilon_{22}\right)$ deformation, in which the stress state is more homogeneous. In addition, the boundary conditions at the crystal surfaces are identical (imposed displacements at the external surface, zero stress at the void surface) and the image forces on the dislocations are equivalent for all slip planes. Thus, the influence of lattice orientation should be minor in the mechanical response of a crystal subjected to uniaxial and biaxial deformation. The stress-strain curves $\left(\sigma_{22}-\right.$ $\epsilon_{22}$ ) of the voided crystal deformed under uniaxial and biaxial deformation are plotted in Fig. 5(a) and (b), respectively. Only one simulation was carried out for each orientation and loading condition because previous studies (Segurado and LLorca, 2009) showed that the scatter in the stress-strain curves due to variations of the distribution of sources under biaxial stress states was much lower than in uniaxial tension. Nevertheless, the mechanical behavior was practically independent of the crystal orientation for the reasons stated above. The similitudes in the mechanisms of deformation can be found in the contour plots of the crystals oriented in the $[\overline{1} 10]$ and $[\overline{1} 11]$ directions under biaxial deformation (Fig. 6 ). The three slip systems were operative for both orientations and plastic deformation was particularly intense around the void due to the associated stress concentration. It is also worth noting that the most active slip system changed periodically around the void depending on their orientation with respect to the stress field induced by the biaxial deformation on the void surface. The differences in the stress state (and in the active slips systems) between uniaxial tension and uniaxial/biaxial deformation also led to differences in void shape. Circular voids adopted an elliptical shape under uniaxial tension (Figs. 3a and 4a) with the longer axis parallel to the loading direction, and remained circular if subjected to an isotropic stress state (Fig. 6).

It should be also noted that the overall features of the stress-strain curves under uniaxial/biaxial deformation were similar to those found under uniaxial tension: the initial elastic region ended with the onset of plastic deformation by the nucleation of dislocations. In addition, the micromechanisms of deformation under uniaxial and biaxial deformation were
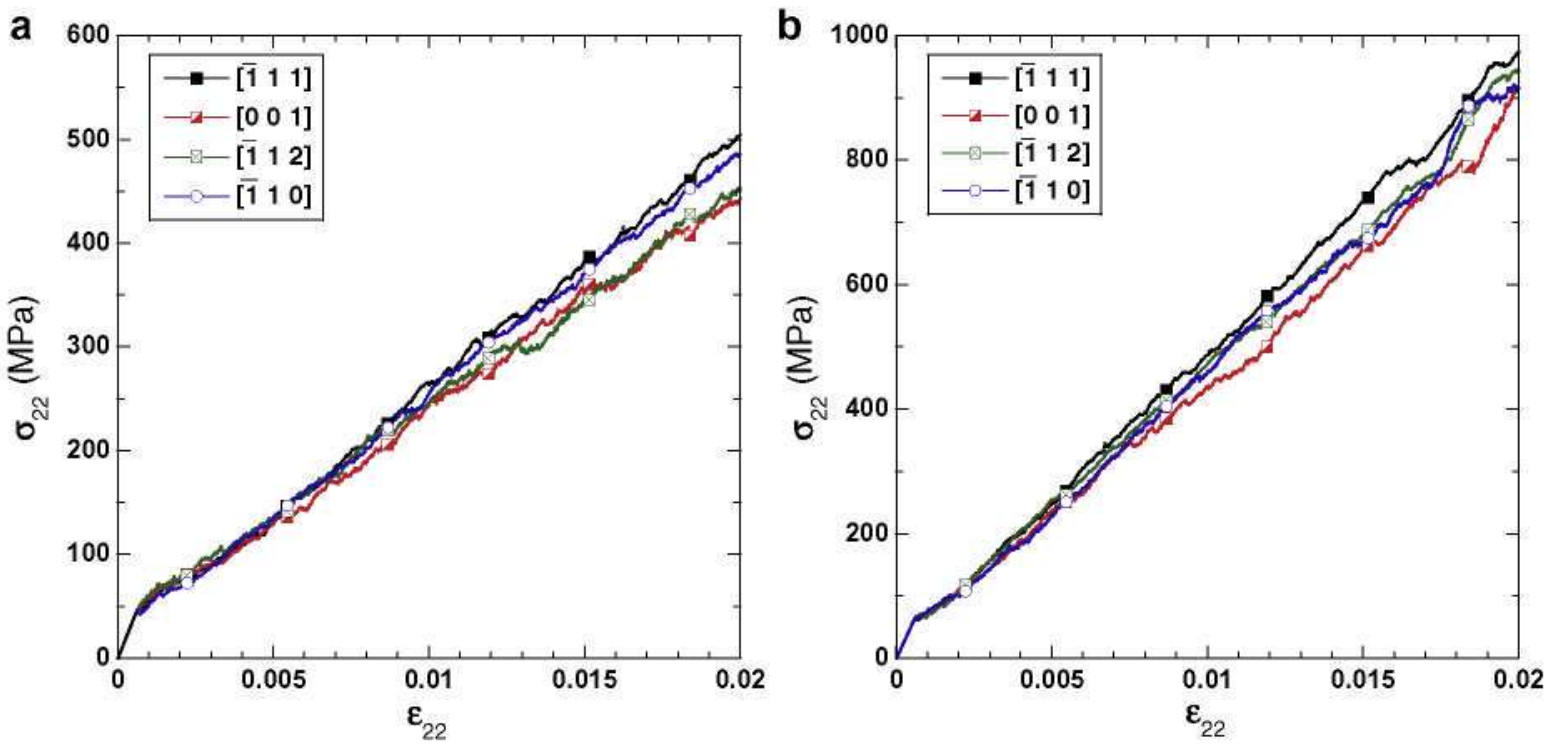

Fig. 5. $\sigma_{22}-\epsilon_{22}$ curves of the voided crystal for different orientations. (a) uniaxial deformation $\left(\epsilon_{11}=0\right)$. (b) Biaxial deformation $\left(\epsilon_{11}=\epsilon_{22}\right)$. 
a

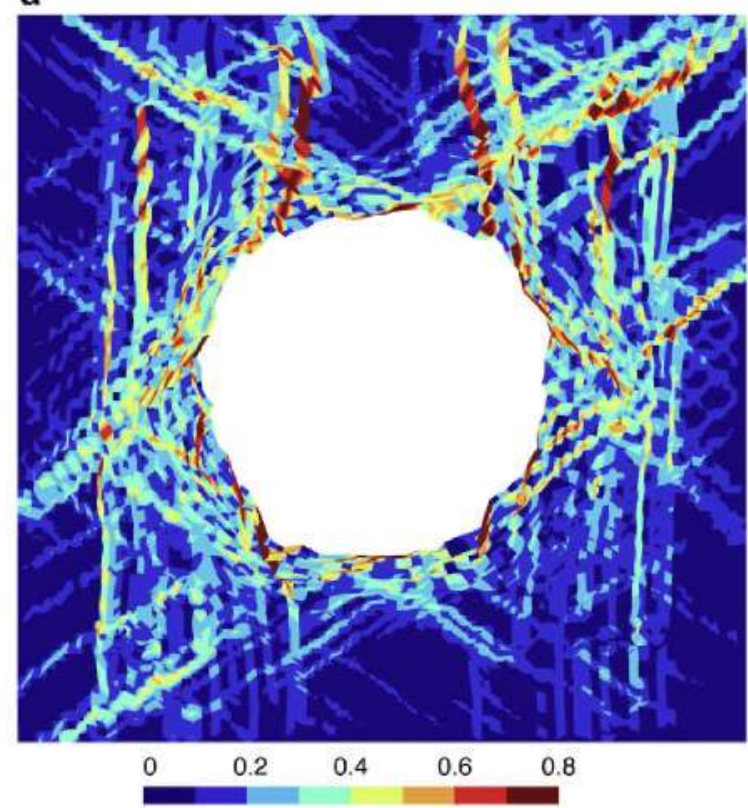

b

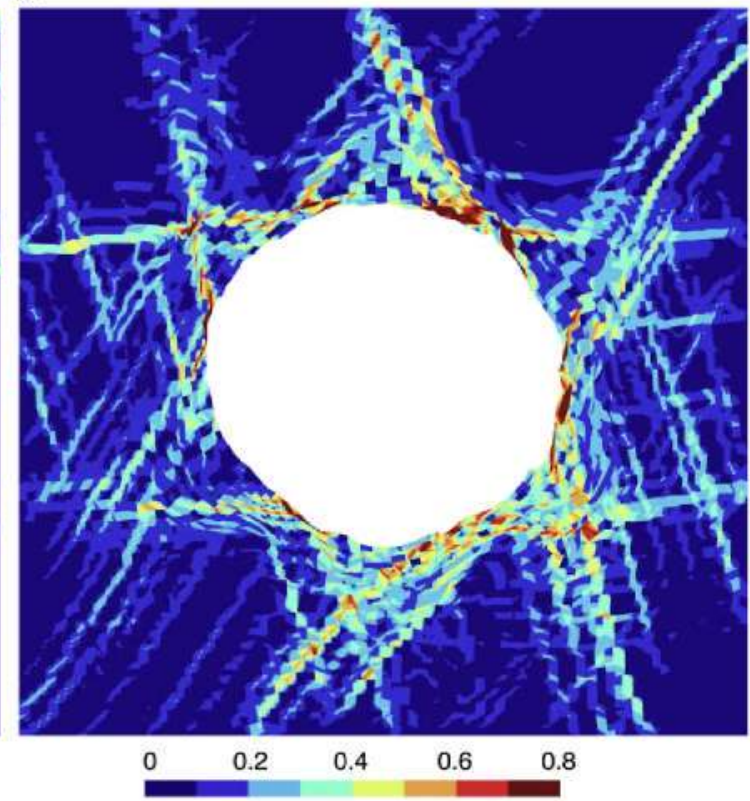

Fig. 6. Contour plot of the total strain in the crystal subjected to biaxial deformation at a far-field strain of $2 \%$. The displacement magnification factor is equal to 3 . (a) $[\overline{1} 10]$ orientation. (b) $[\overline{1} 111]$ orientation.

equivalent to those reported above under uniaxial tension: plastic deformation was localized in particular slip planes of the three available slip systems and hardening was caused by the formation of dislocation pile-ups at the crystal boundaries. However, the strain hardening rate in the plastic regime was much higher compared with uniaxial tension, and this phenomenon was studied in detail in a previous publication (Segurado and LLorca, 2009). Basically, the dramatic increase in hardening rates with triaxiality was due to the synergistic contribution of two factors. The first one is the hydrostatic stress level generated during either uniaxial or biaxial deformation, which increases the overall stress level necessary to nucleate and move dislocations. The second one is the higher dislocation density associated with the activation of all slip systems and not only one or two.

The analyses presented above were carried out under the assumption of small strains, i.e. neglecting changes in geometry of the specimen due to deformation. This is perfectly accurate for the maximum far-field strain attained ( $2 \%$ ) and it can be a reasonable assumption for the crystals loaded under uniaxial tension, in which the maximum strains around the void are in the range 10-15\% (Figs. 3 and 4a). Nevertheless, much larger strains are found around the void in the specimens deformed under uniaxial/biaxial deformation (Fig. 6). These large strains introduce an error in the simulations because the effects of finite deformation-induced lattice rotations and shape changes due to slip on the momentum balance are neglected. The influence of these errors on the mechanical response was analyzed by Deshpande et al. (2005), who simulated the behavior of single crystal of different sizes subjected to unconstrained and constrained uniaxial tension. The mechanical behavior of the single crystals subjected to constrained tension was very similar to the results reported here for voided single crystals: a linear elastic region followed by linear hardening in the plastic region due to the build-up of geometrically necessary dislocations. The constraint imposed by restraining the rotation of the tensile axis resulted in the formation of a kink-like band at $-45^{\circ}$ with respect to the loading axis. (i.e. perpendicular to the original slip direction) and in the development of large bending stresses. These large bending stresses led to the development of lattice rotations and suggested the need for the finitestrain framework. However, simulations carried out under small-strain and finite-strain assumptions led to very similar results. In particular, the dominant deformation micromechanisms and the main features of the stress-strain curves were perfectly captured by the small-deformation analyses. Finite deformation effects (e.g. lattice rotations and shape changes) reduced slightly the predicted hardening rate and gave rise to a tension-compression asymmetry, but did not modified significantly the mechanical response. Thus, it is reasonable to assume that the effect of finite deformations is not critical in our case taking into account the similarities with the problems studied by Deshpande et al. (2005). In addition, the main cause of the differences between large and small-strain formulations (lattice rotations) was minimized in the case of voided crystals subjected to uniaxial/biaxial deformation because the overall stress state in the crystal plane was isotropic.

\subsubsection{Void growth}

Crystal orientation did not only influence the mechanical response of the crystal but the void growth rate. To quantify this effect, the actual area of the void for each crystal orientation was computed from the displacement of the nodes at the void 

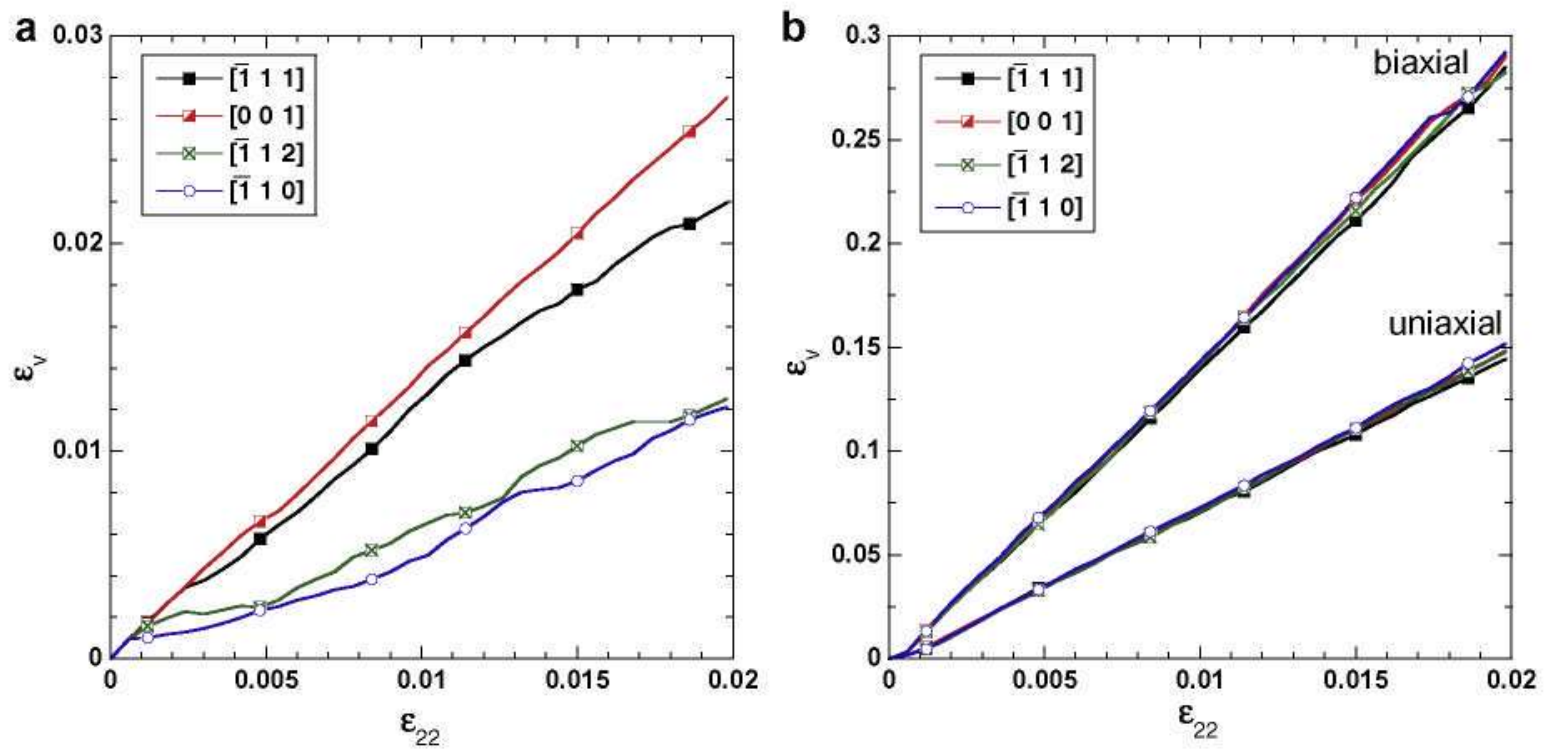

Fig. 7. Evolution of the void area (divided by the initial void area), $\epsilon_{v}$, as a function of the applied strain, $\epsilon_{22}$, for crystals with different lattice orientations. (a) Uniaxial tension $\left(\sigma_{11}=0\right)$. (b) Uniaxial deformation $\left(\epsilon_{11}=0\right)$ and biaxial deformation $\left(\epsilon_{11}=\epsilon_{22}\right)$.

surface during the analyses. The increase in void area divided by the initial void area, $\epsilon_{v}$, is plotted as a function of the applied strain for all the crystal orientations subjected to uniaxial tension (Fig. 7(a)) and uniaxial and biaxial deformation (Fig. $7($ b)). The curves for uniaxial traction are the average of three simulations with different distributions of sources, while uniaxial and biaxial analyses were carried out only once for each orientation.

The physical mechanisms of plastic deformation in uniaxial tension depended on the crystal orientation and this led to changes in growth rates. In general, the hardest orientations ([111] and [001]) presented the highest growth rates while the voids grew more slowly in the softest orientations $([\overline{1} 12]$ and $[\overline{1} 10])$. The differences in growth rates between both groups were of the order of 2, although the overall growth rate under uniaxial tension was small. These results can be explained as follows. Plastic deformation was localized in different slip systems for each orientation and contributed differently to the changes in the crystal and void shape. While the void elongation in the $x_{2}$ direction was similar for all lattice orientations (it was mainly controlled by the imposed deformation along this axis), the deformation of the equator of the void depended on the orientation of the active slip systems and their interaction with the lateral free surfaces. Overall, the [1 11] and [001] crystal orientations which induced higher dislocation densities (and thus stronger crystals) also led to higher void growth rates.

This behavior (stronger crystals led to higher growth rates) was opposite to the one found in our previous investigation on the effect of crystal and void size on the flow stress and void growth rates in single crystals (Segurado and LLorca, 2009). This investigation showed that the smaller the size the higher the flow stress and the lower the void growth rate and this behavior was related to the build-up of elastic stress as the crystal size decreased because the total number of dislocations available in the smallest crystals was limited by crystal size and source density. Thus, the applied strain has to be partially absorbed through elastic deformation, substantially increasing the hardening rate. In addition, the total volumetric strain was the addition of volumetric elastic strains and void growth because the plastic deformation only induces shear strains. Higher elastic strain in smaller specimens reduced the void growth rate accordingly.

As all the orientations presented similar mechanisms of deformation for different orientations under uniaxial and biaxial deformation, void growth rates were also independent of lattice orientation. Growth rates increased rapidly, however, with triaxiality and this was a result of the deformation mode. The total volumetric strain (given by $\epsilon_{11}+\epsilon_{22}$ ) is the addition of volumetric elastic strains and void growth because the plastic deformation only induced shear strains. As the contribution of the volumetric elastic strains to $\epsilon_{11}+\epsilon_{22}$ is limited (and equivalent for all lattice orientations because the stress-strain curves are very close), most of the volumetric strain has to be accommodated by void growth.

It is interesting at this point to compare the results of the DDD simulations with those obtained by Potirniche et al. (2006b) using the finite element method. These authors also reported that lattice orientations did influence the growth rate in uniaxial tension but not in uniaxial or biaxial deformation. In addition, the void growth and strain hardening rates were correlated in crystals loaded in uniaxial tension and the stronger crystals presented higher void growth rates. The ranking of crystal orientations in terms of void growth rates was, however, different to the results presented in Fig. 7. Crystals loaded along the [211] and [001] directions presented the softest response and the lowest void growth rates while orientations $[\overline{1} 11]$ and $[110]$ led to the highest strength and void growth rates. These disparities were caused by the differences in the boundary conditions between both analyses. The periodic boundary conditions used in Potirniche et al. (2006b) to 
simulate the behavior of an infinite regular array of voided single crystals constrained the lateral displacement of crystal which was unconstrained in the DDD simulations. As a result, the pattern of plastic deformation was modified and this influenced the hardening and void growth rates of the crystals. Nevertheless, the boundary conditions used in our DDD model and in their finite element simulations were equivalent under uniaxial and biaxial deformation. Both simulations predicted that the void growth rate was independent of the lattice orientation in these cases.

\subsection{Effect of the number of active slip systems}

The number of active slip systems is another important factor controlling plastic deformation within a single crystal. This effect is examined in this section for a single crystal whose [001] direction was parallel to $x_{2}$-axis $\left(\phi=0^{\circ}\right.$ in Fig. 1). Crystals with one, two or three active slip systems were analyzed. The only active slip direction in the first set of simulations was system (i), which forms an angle of $54.7^{\circ}$ with the $x_{1}$-axis. Systems (i) and (iii) $\left( \pm 54.7^{\circ}\right.$ with $x_{1}$-axis) were chosen in the crystal with two active slip systems. Finally, systems (i), (ii) and (iii) (as in the previous section) were introduced in the crystal with three active slip systems. The source density in the crystal was the same for all the cases $\left(150 \mu \mathrm{m}^{-2}\right)$ and the resulting number of sources was uniformly distributed among the planes of the available slip systems. As in the previous section, simulations with three different source realizations were carried out for the crystals deformed in uniaxial tension while only one realization was used for uniaxial and biaxial deformation.

The stress-strain curves $\left(\sigma_{22}-\epsilon_{22}\right)$ of the crystals deformed under uniaxial tension are plotted in Fig. 8(a) together with the evolution of dislocation density, Fig. 8(b). Each curve is the average of three different realizations. The yield stress was the same for the three cases because all the configurations include the system (i), which has the highest Schmidt factor. After the onset of plastic deformation, the strain hardening rate increased with the number of active slip systems as well as the dislocation density. Nevertheless, the influence of the number of active slip systems on the mechanical response was small although the differences in dislocation densities were noticeable. The higher the number of active slip systems the higher the probability of finding a source on a plane favorably oriented to nucleate a dislocation dipole and crystals with more slip systems presented higher dislocation densities. Nevertheless, the contribution of these dislocations to the hardening of the crystal was different if one or more slip systems were active. In the former, all the dislocations were concentrated in the only available slip system while the dislocation densities in the crystal with three active slip systems at $\epsilon_{22}=2 \%$ were, respectively, 36\%, 26\% and 38\% for systems (i), (ii), and (iii). The Schmidt factor of systems (i) and (iii) was high and the dislocation activity in these planes was maximum, but it is worth noting that system (iii) also had a significant number of dislocations even though its Schmidt factor was 0. Obviously, the contribution of forest dislocations to hardening is higher if all were concentrated in the same slip system and the strength of the crystal with one slip system was only slightly lower than that of those with two or three slip systems.

The influence of the number of active slip systems on the mechanical response was also analyzed in a crystal subjected to uniaxial and biaxial deformation. Only the latter results have been plotted in Fig. 9 for the sake of brevity. They show that the crystals with two or three active slip systems presented very similar stress-strain curves $\left(\sigma_{22}-\epsilon_{22}\right)$ although the dislocation
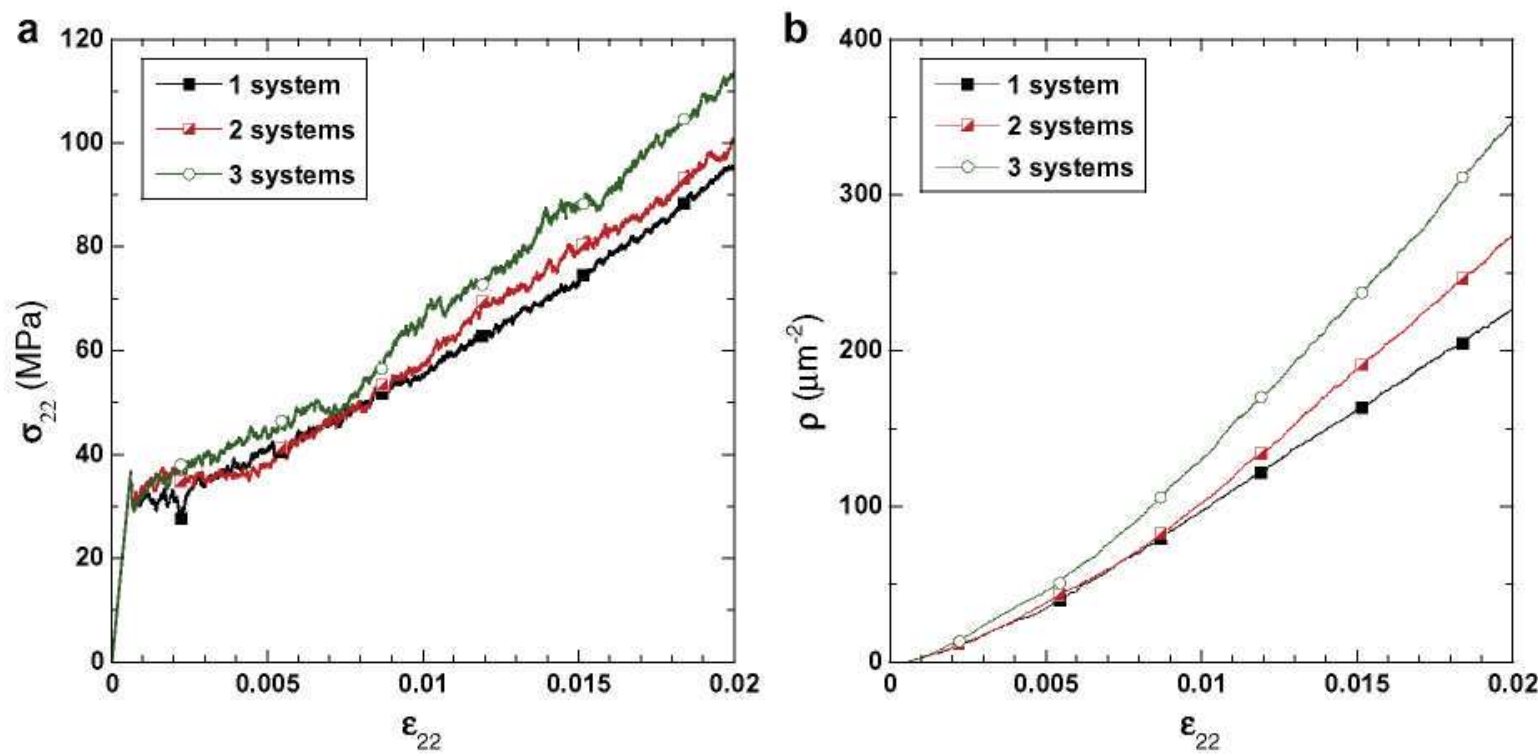

Fig. 8. Averaged $\sigma_{22}-\epsilon_{22}$ curves of the voided crystal with different number of active slip systems subjected to uniaxial tension. (b) Evolution of the dislocation density with the applied strain. 

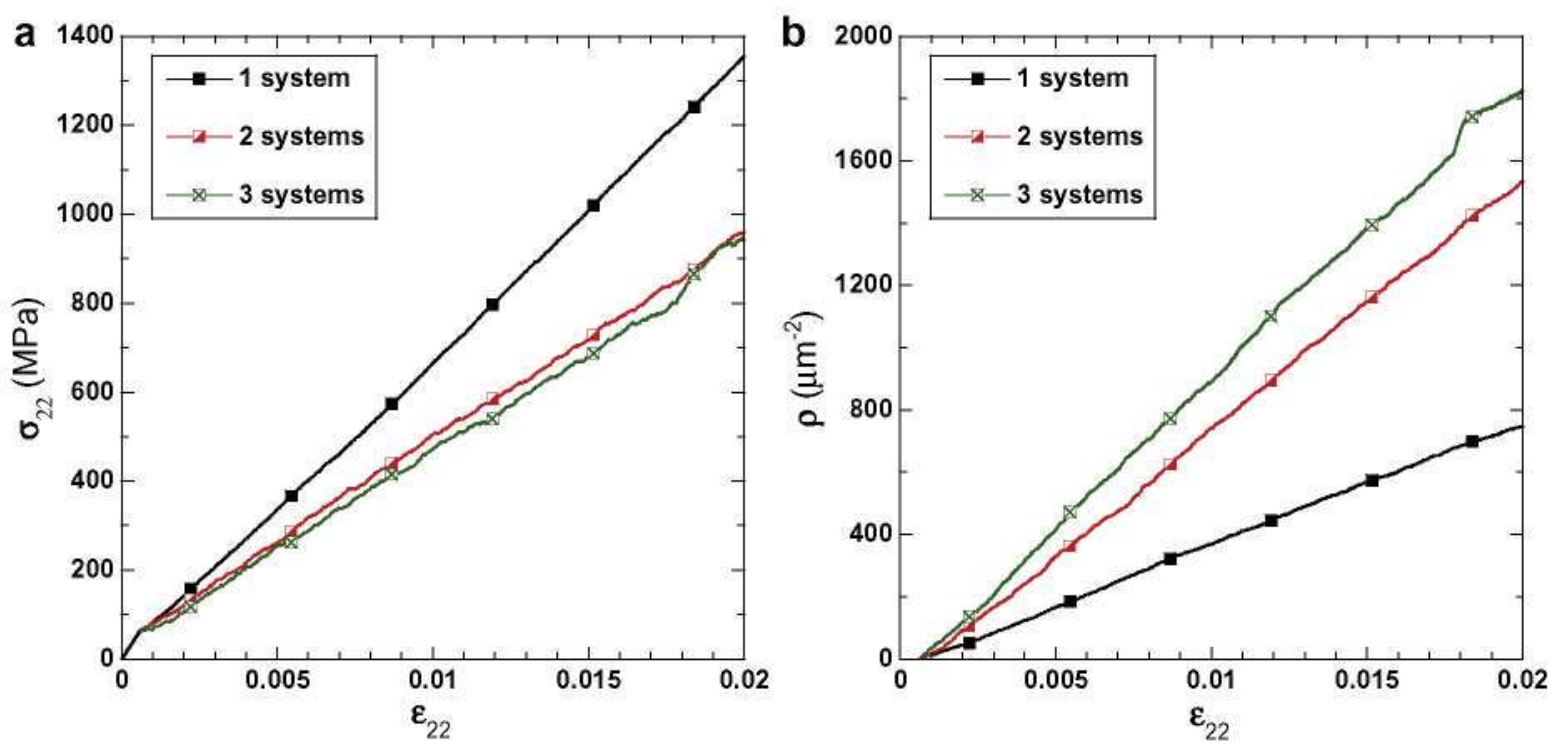

Fig. 9. Averaged $\sigma_{22}-\epsilon_{22}$ curves of the voided crystal with different number of active slip systems subjected to biaxial deformation. (b) Evolution of the dislocation density with the applied strain. The crystal is oriented with the [001] direction parallel to the $x_{2}$-axis.

densities increased with the number of active systems for the reasons stated above. Nevertheless, the crystal with only one active slip system presented the highest hardening rate although the dislocation density was the lowest. Similar results were obtained for the crystals subjected to uniaxial deformation. These differences in the mechanical response between the crystal with one active slip system and those with two or three were also reflected in the dislocation patterns at $\epsilon_{22}=2 \%$, which are plotted in Fig. 10 for the crystal subjected to biaxial deformation. The overall deformation of the crystal was symmetric with respect to the $x_{1}$ - and $x_{2}$-axes and thus there was an equilibrium in the density of positive and negative dislocations to accommodate the crystal deformation without rotation. The "anomalous" response of the voided crystal with only one slip system under uniaxial and biaxial deformation is related to the incompatibility between the symmetric deformation mode imposed by the boundary conditions and the asymmetric plastic deformation induced by the only active slip system. System (i) is oriented at an angle of $54.7^{\circ}$ with the $x_{1}$-axis. Dislocation slip in this plane induces horizontal shear strains which are restricted by the boundary conditions, leading to the development of elastic stresses which significantly increase the hardening rate (Fig. 11a). This mechanism is not operative in crystals in which slip systems (i) and (iii) are active, because the combination of the plastic strains generated by dislocation slip in both systems is compatible with the symmetric deformation pattern imposed by the boundary conditions (Fig. 10b and c). As a result, the hydrostatic stresses and hardening rates were lower (Fig. 11b).

DDD simulations also showed that the most active slip system and the sign of the dislocations changed periodically around the void (Fig. 10). The maximum principal stress at the void surface is the hoop stress, whose modulus is constant under biaxial deformation. The activity of each slip system around the void can be easily determined by computing the projection of the hoop stress on the corresponding slip plane. For instance, systems (i) and (iii) (oriented at $\phi= \pm 54.7^{\circ}$ with $x_{1}$ ) are the best suited for slip at $\phi= \pm 9^{\circ}$ and $\phi= \pm 171^{\circ}$ (Fig. 10a and b), while system (ii) (parallel to $x_{1}$ ) presented the highest Schmidt factor at $\pm 45^{\circ}$ and $\pm 135^{\circ}$. (Fig. $10 \mathrm{c}$ ). As a result, the most active slip system changed periodically around the void. Gan et al. (2006) tested an Al single crystal containing a cylindrical void in uniaxial compression, and the load direction and crystal orientation were equal to ours. The activity of each slip system around the void was determined by measuring the inplane lattice rotation by EBSD, and the results were in good agreement with our DDD simulations for the crystal with three active slip systems. It was found that the most active slip system varied periodically around the void, following the pattern found in Fig. 10c. Thus, dislocation slip along horizontal planes corresponding to system (ii) was dominant at sectors oriented at $\pm 45^{\circ}$ and $\pm 135^{\circ}$, while most of the dislocation emanating from the equator or the pole of the void belonged to systems (i) and (iii).

Finally, the void growth rate for the crystals with a different number of active slips systems was obtained as a function of the applied strain $\epsilon_{22}$. The results are plotted in Fig. 12(a) and (b) for crystals deformed under uniaxial tension and biaxial deformation, respectively. The results for uniaxial deformation were equivalent to those under biaxial deformation (although the overall growth rates were smaller) and are not plotted for the sake of brevity. The void growth rates under uniaxial tension were smaller and the differences among the crystals with one, two or three slips systems were also minimum, following the behavior reported for the stress-strain curves (Fig. 8). Void growth rates under biaxial deformation were much higher and there were important differences between the crystal with one slip system and those with two or three systems, the growth rates being slower in the former. As in the $\sigma_{22}-\epsilon_{22}$ curves (Fig. 9a), these differences were associated to the devel- 

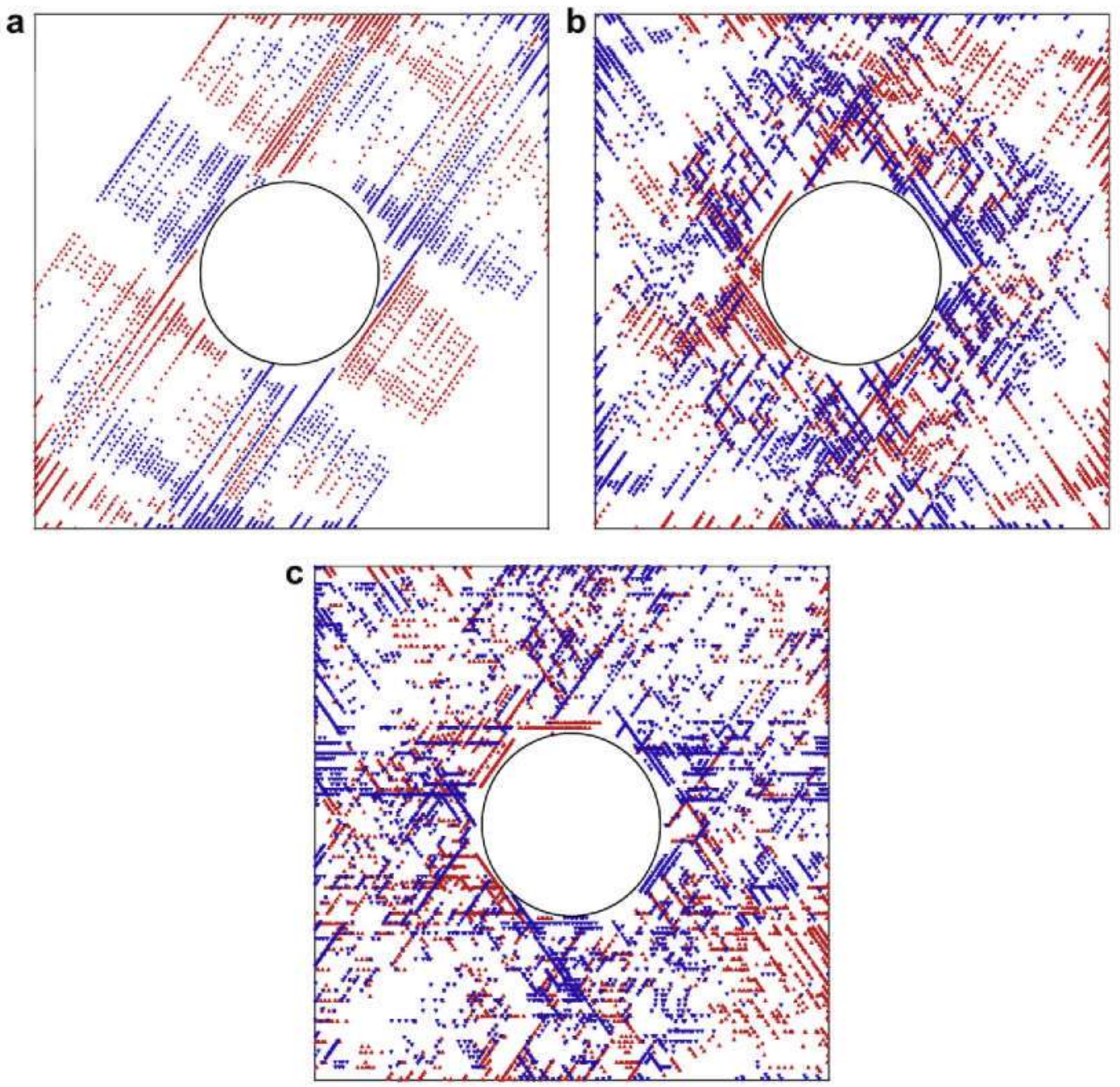

Fig. 10. Dislocation pattern at $\epsilon_{22}=2 \%$ in the voided crystal with different number of active slip systems subjected to biaxial deformation. (a) Active slip system (i). (b) Active slip systems (i) and (iii). (c) Active slip systems (i)-(iii).

opment of large hydrostatic elastic stresses during deformation in the voided crystal with only one slip system. The total volumetric strain (given by $\epsilon_{11}+\epsilon_{22}$ ) is the addition of volumetric elastic strains and void growth because plastic deformation only induces shear strains. As the elastic stresses were higher in the crystal with one slip system, the volumetric deformation of the void has to be smaller.

\section{Concluding remarks}

Discrete dislocation dynamics was used to study the effect of crystal orientation and number of available slip systems on the mechanical behavior of a voided FCC single crystal subjected to plane strain deformation within the (110) crystallographic plane. Results in terms of the stress-strain curves and void growth rates were obtained for three different loading situations, namely uniaxial tension, uniaxial deformation and biaxial deformation. Crystals loaded in uniaxial tension along the [001] and [111] directions were stronger and presented higher growth rates than those deformed in [110] and [112]. These differences among crystal orientations were explained in terms of a number of factors. Low hardening and void growth rates were found in orientations in which the most active slip planes (high Schmidt factor) were in regions where the applied stresses were high and had free surfaces (that attract dislocations) at both ends of the slip planes. However, higher stresses have to be applied if the most active slip planes have to pass above and below the void (precisely the region where the applied stresses are minimum) and finished at the upper or lower crystal surfaces, where the image forces due to the imposed displacements repel the dislocations. Crystal orientation did not influence hardening and void growth when the crystals were loaded under uniaxial or biaxial deformation, although both (hardening and void growth rates) were substantially higher than under uniaxial tension. The stress state was more homogeneous and all the external crystal boundaries had 
a

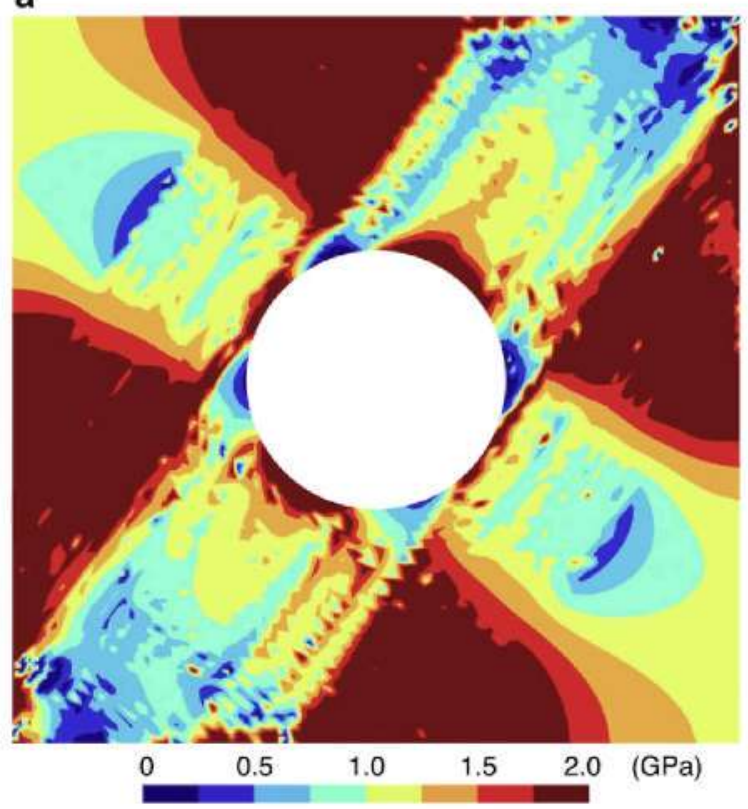

b

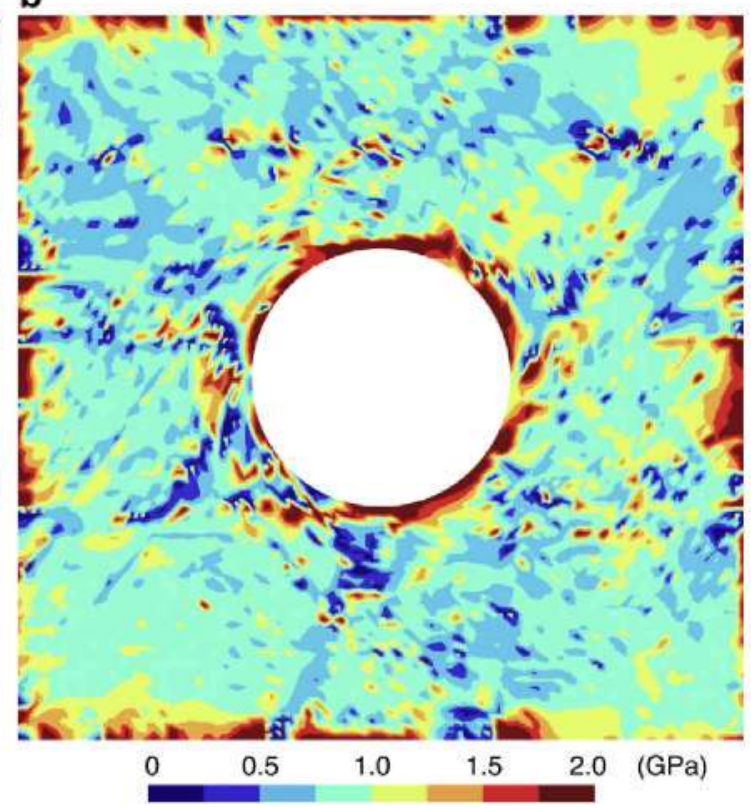

Fig. 11. Contour plot of the hydrostatic stress in the voided crystal subjected to biaxial deformation at a far-field strain of $2 \%$. (a) Only slip system (i) is active. (b) Slip systems (i)-(iii) are active. The crystal is oriented with the [001] direction parallel to the $x_{2}$-axis.
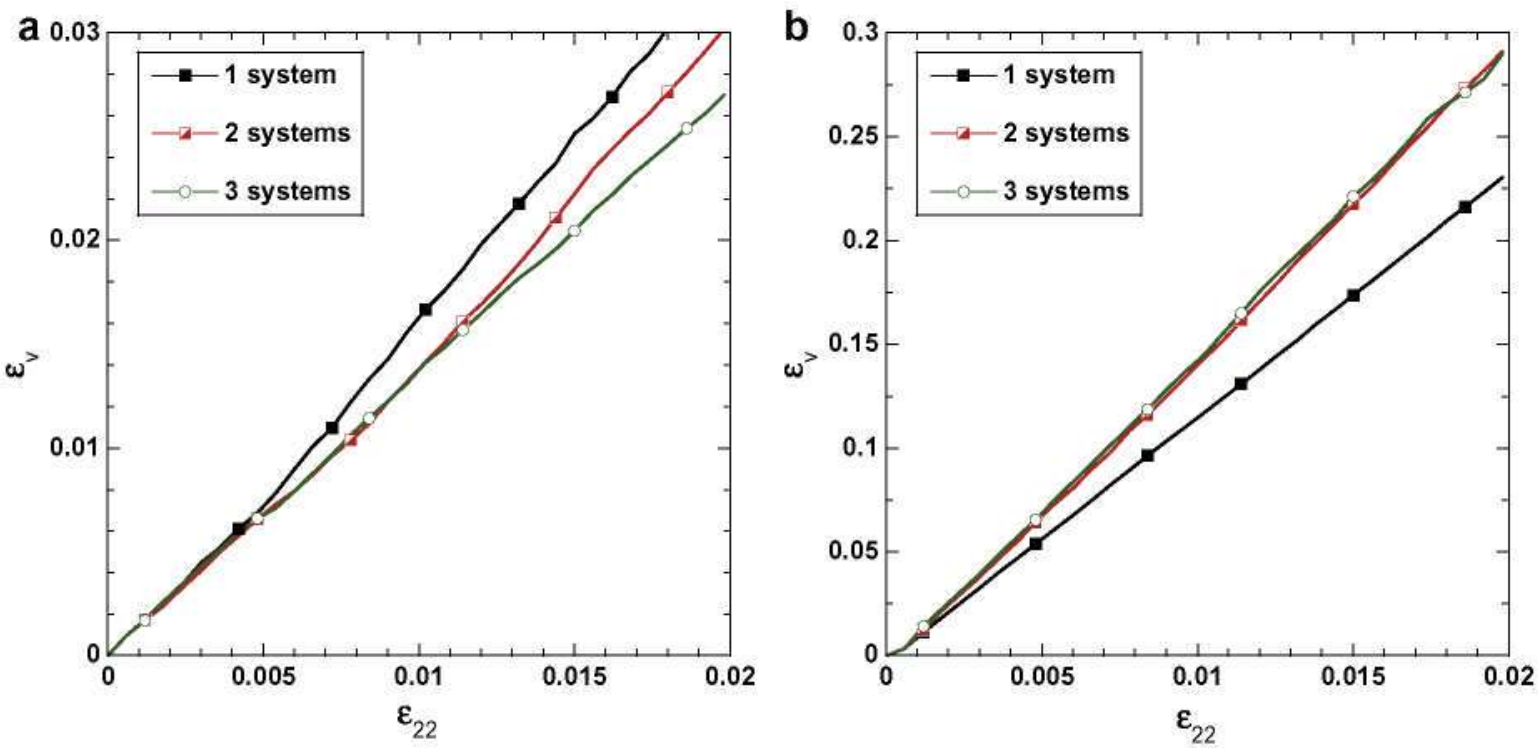

Fig. 12. Evolution of the void area (divided by the initial void area), $\epsilon_{v}$ as a function of the applied strain, $\epsilon_{22}$, for crystals with for crystals with one, two and three active slip systems. (a) Uniaxial tension $\left(\sigma_{11}=0\right)$. (b) Biaxial deformation $\left(\epsilon_{11}=\epsilon_{22}\right)$.

the same boundary conditions (imposed displacements). As a result, neither the orientation nor the location of the slip planes controlled their activity.

The influence of the number of active slip systems $(1,2$ or 3$)$ was studied in crystal oriented with the [001] direction parallel to the $x_{2}$-axis. The hardening and void growth rates were similar under uniaxial tension, regardless of the number of active slip systems, because the lateral deformation was unconstrained and it was possible to accommodate the plastic strain even if only one slip system was available. However, this was not the case under uniaxial and biaxial deformation, where the overall deformation has to be symmetric. The incompatibility between the symmetric deformation mode imposed by the boundary conditions and the asymmetric plastic deformation induced by the only active slip system led to the development of high hydrostatic elastic stresses. They increased the strain hardening rate and reduced the void growth rate as 
compared with crystals with two or three active slip systems, іл which dislocation slip promoted a symmetric deformation pattern compatible with the boundary conditions.

It should be finally noted that computational power limits the current DDD simulation capabilities to high strain rates. However, it is expected that the mechanical response and void growth rates predicted by DDD are representative of the actual behavior at lower strain rates. The mechanical response of the voided single crystals showed a marked bilinear behavior іл all cases. The yield stress, which indicated the onset of plastic deformation, was controlled by the critical resolved shear stress to nucleate a dipole and the Schmidt factor corresponding to the most favorable slip system. Linear hardening during plastic flow was induced by the formation of dislocation pile-ups at the crystals boundaries as well as by forest hardening. which hindered the mobility of dislocations and reduced the activity of the sources through the generation of a back stress. These mechanisms - controlling the onset of plastic flow and linear hardening - are fairly independent of the loading rate and the mechanical response should not be essentially modified by this factor.

\section{Acknowledgments}

This investigation was supported by the Ministerjo de Eduación y Ciencia de España through the grants MAT 2006-2602 and MAT 2009-14396 and by the Comunidad de Madrid through the program ESTRUMAT-CM (reference MAT/0077).

\section{References}

Borg. U., Niordson, C.F., Kysar, J.W., 2008. Size effects on void growth in single crystals with distributes voids. International Journal of Plasticity $24,688-701$. Chng, A.C., ODay, M.P., Curtin, WA., Tay, A.0.A., Lim, K.M., 2006. Fracture in confined thin films: a discrete dislocation study. Acta Materialia 54, 1017-1027.

Deshpande, V.S., Needleman, A., Van der Giessen, E., 2005. Plasticity size effects in tension and compression of single crystals. Journal of the Mechanics and Physics of Solids 53, 2661-2691.

Essmann, U., Mughrabi, H., 1979. Annihilation of dislocations during tensile and cyclic deformation and limits of dislocation densities. Philosophical Magazine A 40, 731-756.

Fleck, N.A., Muller, G.M., Ashby, M.F., Hutchinson, J.W., 1994. Strain gradient plasticity: theory and experiment. Acta Metallurgica et Materialia 42, 457-487.

Gan, Y.X., Kysar, J.W., Morse. T.l., 2006. Cylindrical void in a rigid-ideally plastic single crystal ii: experiments and simulations. International Journal of Plasticity 22, 39-72.

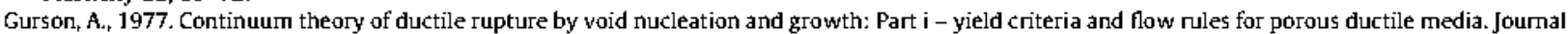
of Engineering Materials and Technology 9, 2-15.

Hom, C.L., McMeeking, R.M., 1989. Void growth in elastic-plastic materials. Journal of Applied Mechanics 56, 309-317.

Huang, M., Li, Z., Wang C., 2007. Discrete dislocation dynamics modelling of microvoid growth and its intrinsic mechanism in single crystals. Acta Materialia 55, 1387-1396.

Hussein, M., Borg, U., Niordson, C.F., Deshpande, V.S., 2008. Plasticity size effects in voided crystals. Journal of the Mechanics and Physics of Solids 56, 114131.

Koplik, JA.N., 1988. Void growth and coalescence in porous plastic solids. International Journal of Solids and Structures 11, 835-853.

Kysar, J.W., Gan, Y.X., Mendez-Arzuza, G., 2005. Cylindrical void in a rigid-ideally plastic single crystal. Part i: Anisotropic slip line theory solution for facecentered cubic crystals. International Joumal of Plasticity 21, 1481-1520.

Li, Z., Steinmann, P., 2006. Rve-based studies on the coupled effects of void size and void shape on yield behavior and void growth at micron scales. lnternational Journal of Plasticity 22, 1195-1216.

Liu, B., Huang, Y., Li, M., Hwang, K.C., Liu, C., 2005. A study of the void size effect based on the Taylor dislocation model. International Journal of Plasticity 21 , 2107-2122.

Liu, B., Qju, X., Huang, Y., Hwang, K.C., Li, M., Liu, C., 2003. The size effect on void growth in ductile materials. Journal of the Mechanics and Pliysics of Solids $51,1171-1187$.

Liu, W.H., Zhang. X.M., Tang. J.G., Du, Y.X., 2007. Simulation of void growth and coalescence behavior with 3D crystal plasticity. Conputational Materials Science 40, 130-139.

Mott, N., 1952. A theory of work-hardening of metal clystals. Philosophical Magazine 43. 1151-1178.

Motz, C., Schöberl, T., Pippan, T.R., 2005. Mechanical properties of micro-sized copper bending beams machined by the focused ion beam technique. Acta Materialia 53, 4279-4296.

Potirniche, G., Horstemeyer, M., Wagner, G., Gullett, P., 2006a. A molecular dynamics study of void growth and coalescence in single crystal nickel. International Journal of Plasticity 22, 257-258.

Potirniche, G.P., Hearndon, J.L., Horstemeyer. M.F., ling, X.W., 2006b. Lattice orientation eflects on void growth and coalescence in fcc single crystals. International Journal of Plasticity 22, 921-942.

Quinn, D., Connolly, P., Howe, M., McHugh, P., 1995. Simulation of void growth in WC-Co hardmetals using crystal plasticity theory. International Journal of Mechanical Sciences 39, 173-183.

Rice, J., Tracey, D., 1969. On the ductile enlargement of voids in triaxial stress fields. Journal of the Mechanics and Physics of Solids 17, $201-207$.

Rice. J.R., 1987. Tensile clack tip fields in elastic-ideally plastic materials. Mechanics of Materials 6. 317-335.

Romero, l., Segurado. J., Llorca, J., 2008. Dislocation dynamics in non-convex domains using finite elements with embedded discontinuities. Modelling and Simulation in Materials Science and Engineering 16, 035008.

Segurado. J., LLorca. J., 2009. An analysis of the size effect on void growth in single clystals using discrete dislocation dynamics. Acta Materialia 57, 14271436.

Segurado, J., Lorca, J., Romero, 1., 2007. Computational issues in the simulation of two-dimensional discrete dislocation dynamics. Modelling and Simulation in Materials Science and Engineering 15, 361-375.

Srinivasan, K., Huang, Y., Kolednik, 0 ., Siegmund, T., 2008. The size dependence of micro-toughness in ductile fracture. Journal of the Mechanics and Physics of Solids 56, 2707-2726.

Taylor, G.I. 1934. The mechanisms of plastic deformation in clystals. Part I. Theoretical. Proceedings of the Royal Sociecy (London) A145, 362-387.

Taylor, G.I., 1938. Plastic strain in metals. Joumal of the Institute of Metals 62, 307-324.

Traiviratana, S., Bringa, E.M., Benson, D.,. Meyers, M.A., 2008. Void growth in metals: atomistic calculations. Acta Materialia 56. 3874-3886.

Tvergaard, V., 1990. Material failure by void growth to coalescence. Advances in Applied Mechanics 27, 83-151.

Uchic. M.D., Diniduk. D.M., Florando. J.N., Nix. W.D., 2004. Sample dimensions influence strength and crystal plasticicy. 5cience 305. 986-989.

Van der Giessen, E. Needleman, A., 1995. Discrete dislocation plasticity: a simple planar model. Modelling and Simulation in Materials Science and Engineering 3, 689-735.

Worswick, M.J., Pick, R.J., 1990. Void growth and constitutive softening in a periodically voided solid. Journal of the Mechanics and Physics of 5olids 38 , 601625. 